\title{
$\alpha$-Aminoallylation of Aldehydes with Ammonia: Stereoselective Synthesis of Homoallylic Primary Amines
}

\author{
Masaharu Sugiura, Keiichi Hirano and Shū Kobayashi* \\ Graduate School of Pharmaceutical Sciences, The University of Tokyo \\ Hongo, Bunkyo-ku, Tokyo 113-0033, Japan
}

\section{Supporting Information}

Table of Contents

Experimental Details and Physical Data

General Methods

Chemicals

General procedures for $\alpha$-aminoallylation of aldehydes 1a-l (Table 1)

Diastereoselective $\alpha$-aminoallylation of $(S)$-1m (Scheme 2)

Diastereoselective $\alpha$-aminoallylation of $(2 S, 3 S)$-1n (Scheme 2)

Transformation of Boc-3n to a L-acosamine derivative (Scheme 2)

$\alpha$-Aminocrotylation of aromatic aldehydes (Scheme 3)

Synthesis of alloisoleucine (Scheme 4)

Enantioselective $\alpha$-aminoallylation of benzaldehyde (Scheme 5)

Detection of an $\mathrm{N}$-unsubstituted imine by ${ }^{1} \mathrm{H}$ NMR analysis

References and notes
Pages

S-1

S-1

S-2

S-2

S-6

S-8

S-9

S-9

S-10

S-11

S-11

S-12

\section{Experimental Details and Physical Data}

General Methods: Melting points are uncorrected. ${ }^{1} \mathrm{H}$ and ${ }^{13} \mathrm{C}$ NMR spectra were recorded on a JEOL JNM-LA300 or JNM-LA400 spectrometer in $\mathrm{CDCl}_{3}$ unless otherwise noted. Tetramethylsilane (TMS) served as internal standard $(\delta=0)$ for ${ }^{1} \mathrm{H}$ NMR and $\mathrm{CDCl}_{3}$ was used as internal standard $(\delta=77.0)$ for ${ }^{13} \mathrm{C}$ NMR. High-resolution electron impact mass spectra (HR-EIMS) were measured with JEOL JMX-SX-102A mass spectrometer. High-resolution electrospray ionization mass spectra (HR-ESIMS) were measured with BRUKER DALTONICS BioTOF II mass spectrometer. Preparative thin-layer chromatography (PTLC) 
was carried out using Wakogel B-5F. Ethanol was distilled from sodium and stored over $3 \AA$ MS. Distilled water was employed for the aqueous reactions. All other solvents were purified based on standard procedures.

Chemicals: Aldehydes 1a and 1d-k were purified by distillation prior to use. Aldehydes $\mathbf{1 b}$ and 1c were purified by recrystallization from aqueous ethanol and ethanol, respectively. Glyoxylic acid monohydrate (11) was purchased from Tokyo Kasei Kogyo Co., Ltd. and used without further purification. Aldehyde $(S)-\mathbf{1} \mathbf{m}^{1}$ and $(2 S, 3 S)-\mathbf{1} \mathbf{n}^{2}$ were prepared according to the literature procedures. Allylboronate $\mathbf{2},{ }^{3}(E)$ - and $(Z)$-crotylboronate $\mathbf{5},{ }^{4}$ and chiral allylboronate $\mathbf{7}^{5}$ were prepared according to the literatures. Liquid ammonia $(99.999 \%$, Nippon Sanso Corporation) and aqueous ammonia (28.0-30.0 wt\%, Wako Pure Chemical Industries, Ltd.) were used without purification. Palladium on carbon (5 wt\%, PH-type, wet, Kawaken Fine Chemicals Co., Ltd.) were used for hydrogenation. All other chemicals were purified based on standard procedures.

\section{General procedures for $\alpha$-aminoallylation of aldehydes 1a-I (Table 1)}

Method A (entries 1 and 3-9): To a dry two-neck flask with an argon balloon and a rubber septum cooling at $-78{ }^{\circ} \mathrm{C}$, gaseous ammonia was introduced via a needle to obtain liquid ammonia (ca. $0.5 \mathrm{~mL})$. After addition of ethanol $(0.5 \mathrm{~mL})$, aldehyde $1(0.5 \mathrm{mmol})$ was added to ammonia at $-78{ }^{\circ} \mathrm{C}$ and rinsed with ethanol $(0.5 \mathrm{~mL})$. In the case of $\mathbf{1 b}$ and $\mathbf{1 c}$ (solid aldehydes), aldehyde was first placed in the flask before addition of liquid ammonia (ca. 0.5 $\mathrm{mL})$ and ethanol $(1.0 \mathrm{~mL})$. The mixture was warm to $-10{ }^{\circ} \mathrm{C}$ and was stirred at that temperature for $2 \mathrm{~h}$. During that, excess ammonia was vaporized in the balloon. Allylboronate $2(0.6 \mathrm{mmol})$ in ethanol $(0.5 \mathrm{~mL})$ was then added dropwise to the solution. Colorless precipitates were formed on addition of 2 . The mixture was stirred at $-10{ }^{\circ} \mathrm{C}$ for 3 $\mathrm{h}$ and at $\mathrm{rt}$ for $1 \mathrm{~h}$. The balloon and the septum were then removed to vaporize most of ammonia and $1 \mathrm{~N}$ aqueous $\mathrm{HCl}$ was added to acidify the solution ( $\mathrm{pH}$ ca. 1). The mixture was washed with diethyl ether (3x), alkalized with $6 \mathrm{~N}$ aqueous $\mathrm{NaOH}$ (pH ca. 10) and extracted with dichloromethane $(3 \times)$. The dichloromethane layers were dried over anhydrous $\mathrm{Na}_{2} \mathrm{CO}_{3}$, filtered and evaporated to give almost pure amine 3. The crude product was purified by preparative TLC (hexane/isopropylamine $=10 / 1$ ) to give amine 3. Meanwhile, non-basic components including alcohol $\mathbf{4}$ was obtained from the ethereal extracts. The extracts were 
dried over anhydrous $\mathrm{MgSO}_{4}$, filtered and concentrated in vacuo. The yield of alcohol 4 was estimated by ${ }^{1} \mathrm{H}$ NMR analysis using 1,2,4,5-tetramethylbenzene as an internal standard.

Method B (entries 2 and 13): Aldehyde 1a or $11(0.5 \mathrm{mmol})$ in 28-30 wt\% aqueous ammonia (0.74 mL, ca. 20 equiv) and ethanol $(1 \mathrm{~mL})$ was stirred at $\mathrm{rt}$ for $30 \mathrm{~min}$. Allylboronate $2(0.6 \mathrm{mmol})$ was added to the solution and rinsed with ethanol $(0.5 \mathrm{~mL})$. The mixture was stirred at $\mathrm{rt}$ for $2 \mathrm{~h}$ (entry 2) or $3 \mathrm{~h}$ (entry 13) and worked up in the same way as Method A for the reaction of 1a (entry 2). In the case of glyoxylic acid (11) (entry 3), the reaction mixture was concentrated in vacuo and the residue was charged on a cationic ion exchange resin column (DOWEX 50W-X2, 50-100 mesh, $\mathrm{H}^{+}$form; ca. $7 \mathrm{~g} /$ wet) with water. After washing with a sufficient amount of water, the product was eluted with $0.2 \mathrm{~N}$ aqueous ammonia. The fractions were checked by silica gel TLC plates without development using $0.1 \mathrm{wt} \%$ ninhydrine in acetone/water $(80 / 20 \mathrm{v} / \mathrm{v} \%)$. Fractions including product 31 were collected and concentrated to dryness.

Method C (Table 1, entries 10-12): Allylboronate $2(0.6 \mathrm{mmol})$ in ethanol $(0.5 \mathrm{~mL})$ was added to liquid ammonia (ca. $0.5 \mathrm{~mL}$, see Method A) at $-78{ }^{\circ} \mathrm{C}$ and rinsed with ethanol $(0.5 \mathrm{~mL})$. Colorless precipitates were formed during addition of 2 . After being stirred at $\mathrm{rt}$ for $30 \mathrm{~min}$, aldehyde $1(0.5 \mathrm{mmol})$ was added to the suspension and rinsed with ethanol $(0.5$ $\mathrm{mL}$ ). The mixture was stirred at $\mathrm{rt}$ for $2 \mathrm{~h}$ and worked up in the same way as Method A.

1-Phenylbut-3-en-1-amine (3a): ${ }^{6}$ 84\% (Method A), 80\% (Method B); ${ }^{1} \mathrm{H}$ NMR (300 MHz, $\left.\mathrm{CDCl}_{3}\right) \delta$ 7.34-7.21 (m, 5H), 5.76 (dddd, $\left.J=17.0,10.1,8.0,6.5 \mathrm{~Hz}, 1 \mathrm{H}\right), 5.05-5.16(\mathrm{~m}, 2 \mathrm{H})$, $3.99(\mathrm{dd}, J=8.0,5.3 \mathrm{~Hz}, 1 \mathrm{H}), 2.52-2.42(\mathrm{~m}, 1 \mathrm{H}), 2.41-2.30(\mathrm{~m}, 1 \mathrm{H}), 1.55(\mathrm{brs}, 2 \mathrm{H}) ;{ }^{13} \mathrm{C} \mathrm{NMR}$ $\left(75 \mathrm{MHz}, \mathrm{CDCl}_{3}\right) \delta 145.8,135.4,128.4,127.0,126.3,117.6,55.4,44.2$; HR-ESIMS calcd for $\mathrm{C}_{10} \mathrm{H}_{14} \mathrm{~N}\left(\mathrm{M}+\mathrm{H}^{+}\right)$148.1126, found 148.1126.

1-(4-Nitrophenyl)but-3-en-1-amine (3b): ${ }^{6}$ 96\% (Method A); ${ }^{1} \mathrm{H}$ NMR (400 MHz, $\mathrm{CDCl}_{3}$ ) $\delta 8.14$ (apparent d, $J=8.8 \mathrm{~Hz}, 2 \mathrm{H}$ ), 7.50 (apparent d, $J=8.8 \mathrm{~Hz}, 2 \mathrm{H}), 5.69$ (dddd, $J=15.9$, 9.6, 7.9, $6.5 \mathrm{~Hz}, 1 \mathrm{H}), 5.13-5.06(\mathrm{~m}, 2 \mathrm{H}), 4.12(\mathrm{dd}, J=7.8,5.4 \mathrm{~Hz}, 1 \mathrm{H}), 2.43$ (dddt, $J=13.6$, 6.5, 5.4, $1.2 \mathrm{~Hz}, 1 \mathrm{H}), 2.32$ (ddd, $J=13.6,7.9,7.8 \mathrm{~Hz}, 1 \mathrm{H}), 1.59$ (brs, $2 \mathrm{H}) ;{ }^{13} \mathrm{C}$ NMR (100 $\left.\mathrm{MHz}, \mathrm{CDCl}_{3}\right) \delta 153.3,147.0,134.2,127.3,123.6,118.6,54.8,44.1$; HR-ESIMS calcd for $\mathrm{C}_{10} \mathrm{H}_{13} \mathrm{~N}_{2} \mathrm{O}_{2}\left(\mathrm{M}+\mathrm{H}^{+}\right)$193.0977, found 193.0973.

1-(4-Bromophenyl)but-3-en-1-amine (3c): ${ }^{7}$ 92\% (Method A); ${ }^{1} \mathrm{H}$ NMR (400 MHz, $\mathrm{CDCl}_{3}$ ) $\delta 7.45$ (apparent d, $J=8.5 \mathrm{~Hz}, 2 \mathrm{H}$ ), 7.23 (apparent d, $J=8.5 \mathrm{~Hz}, 2 \mathrm{H}), 5.73$ (dddd, $J=17.1$, 
$10.3,8.0,6.4 \mathrm{~Hz}, 1 \mathrm{H}), 5.14-5.08(\mathrm{~m}, 2 \mathrm{H}), 3.97(\mathrm{dd}, J=7.8,5.6 \mathrm{~Hz}, 1 \mathrm{H}), 2.46-2.38(\mathrm{~m}, 1 \mathrm{H})$, 2.36-2.27 (m, 1H), 1.54 (brs, $2 \mathrm{H}) ;{ }^{13} \mathrm{C} \mathrm{NMR}\left(100 \mathrm{MHz}, \mathrm{CDCl}_{3}\right) \delta \quad 144.8,134.9,131.4,128.1$, 120.6, 118.0, 54.8, 44.1; HR-ESIMS calcd for $\mathrm{C}_{10} \mathrm{H}_{13} \mathrm{BrN}\left(\mathrm{M}+\mathrm{H}^{+}\right)$226.0231, found 226.0234. 1-(4-Methoxyphenyl)but-3-en-1-amine (3d): ${ }^{6} 91 \%$ (Method A); ${ }^{1} \mathrm{H}$ NMR (300 MHz, $\left.\mathrm{CDCl}_{3}\right) \delta 7.26$ (apparent d, $\left.J=8.6 \mathrm{~Hz}, 2 \mathrm{H}\right), 6.87$ (apparent d, $\left.J=8.6 \mathrm{~Hz}, 2 \mathrm{H}\right), 5.75$ (dddd, $J=$ 17.2, 10.1, 7.9, $6.3 \mathrm{~Hz}, 1 \mathrm{H}), 5.16-5.04(\mathrm{~m}, 2 \mathrm{H}), 3.95$ (dd, $J=7.9,5.5 \mathrm{~Hz}, 1 \mathrm{H}), 3.80(\mathrm{~s}, 3 \mathrm{H})$, 2.44 (dddt, $J=13.7,6.3,5.5,1.4 \mathrm{~Hz}, 1 \mathrm{H}), 2.34$ (dddt, $J=13.7,7.9,7.9,0.9 \mathrm{~Hz}, 1 \mathrm{H}), 1.54$ (brs, $2 \mathrm{H}) ;{ }^{13} \mathrm{C} \mathrm{NMR}\left(75 \mathrm{MHz}, \mathrm{CDCl}_{3}\right) \delta 158.5,138.0,135.6,127.3,117.5,113.7,55.3,54.8,44.3$; HR-ESIMS calcd for $\mathrm{C}_{11} \mathrm{H}_{16} \mathrm{NO}\left(\mathrm{M}+\mathrm{H}^{+}\right)$178.1226, found 178.1225 .

2-(1-Aminobut-3-enyl)phenol (3e): 76\% (Method A); ${ }^{1} \mathrm{H}$ NMR (400 MHz, $\left.\mathrm{CDCl}_{3}\right) \delta 7.15$ (ddd, $J=8.1,7.3,1.7 \mathrm{~Hz}, 1 \mathrm{H}), 6.96(\mathrm{dd}, J=7.6,1.7 \mathrm{~Hz}, 1 \mathrm{H}), 6.84(\mathrm{dd}, J=8.1,1.2 \mathrm{~Hz}, 1 \mathrm{H})$, 6.78 (ddd, $J=7.3,7.3,1.2 \mathrm{~Hz}, 1 \mathrm{H}), 5.78$ (dddd, $J=16.8,10.3,8.8,5.8 \mathrm{~Hz}, 1 \mathrm{H}$ ), 5.24-5.17 (m, $2 \mathrm{H}), 4.13(\mathrm{dd}, J=9.0,4.9 \mathrm{~Hz}, 1 \mathrm{H}), 2.57-2.43(\mathrm{~m}, 2 \mathrm{H}), \mathrm{NH}_{2}, \mathrm{OH}$ (unassignable); ${ }^{13} \mathrm{C}$ NMR $\left(75 \mathrm{MHz}, \mathrm{CDCl}_{3}\right) \delta 157.7,134.7,128.5,127.7,126.7,119.0,118.9,117.3,55.4,41.5$; HREIMS calcd for $\mathrm{C}_{10} \mathrm{H}_{13} \mathrm{NO}\left(\mathrm{M}^{+}\right)$163.0997, found 163.1001.

1-(Pyridin-2-yl)but-3-en-1-amine (3f): ${ }^{8} 85 \%$ (Method A); ${ }^{1} \mathrm{H}$ NMR (300 $\mathrm{MHz}, \mathrm{CDCl}_{3}$ ) $\delta 8.56(\mathrm{ddd}, J=4.9,1.8,1.1 \mathrm{~Hz}, 1 \mathrm{H}), 7.65(\mathrm{ddd}, J=7.9,7.5,1.8 \mathrm{~Hz}, 1 \mathrm{H}), 7.31(\mathrm{~d}, J=7.9 \mathrm{~Hz}$, 1H), $7.16(\mathrm{ddd}, J=7.5,4.9,1.1 \mathrm{~Hz}, 1 \mathrm{H}), 5.77$ (dddd, $J=17.0,10.2,7.9,6.4 \mathrm{~Hz}, 1 \mathrm{H}), 5.16-$ $5.06(\mathrm{~m}, 2 \mathrm{H}), 4.06(\mathrm{dd}, J=7.9,5.3 \mathrm{~Hz}, 1 \mathrm{H}), 2.60(\mathrm{dddt}, J=13.7,6.4,5.3,1.4 \mathrm{~Hz}, 1 \mathrm{H}), 2.41$ (dddt, $J=13.7,7.9,7.9,1.0 \mathrm{~Hz}, 1 \mathrm{H}), 1.88$ (brs, $2 \mathrm{H}) ;{ }^{13} \mathrm{C} \mathrm{NMR}\left(75 \mathrm{MHz}, \mathrm{CDCl}_{3}\right) \delta$ 164.0, 149.1, 136.4, 135.1, 121.9, 120.9, 117.7, 56.5, 43.2; HR-ESIMS calcd for $\mathrm{C}_{9} \mathrm{H}_{13} \mathrm{~N}_{2}\left(\mathrm{M}+\mathrm{H}^{+}\right)$ 149.1079, found 149.1080 .

1-(Thiophen-2-yl)but-3-en-1-amine (3g): ${ }^{9}$ 77\% (Method A); ${ }^{1} \mathrm{H}$ NMR (400 MHz, $\mathrm{CDCl}_{3}$ ) $\delta 7.19(\mathrm{dd}, J=5.0,1.4 \mathrm{~Hz}, 1 \mathrm{H}), 6.96-6.91(\mathrm{~m}, 2 \mathrm{H}), 5.79$ (dddd, $J=17.1,10.2,7.8,6.4 \mathrm{~Hz}$, $1 \mathrm{H}), 5.18-5.10(\mathrm{~m}, 2 \mathrm{H}), 4.28(\mathrm{dd}, J=7.9,5.2 \mathrm{~Hz}, 1 \mathrm{H}), 2.61-2.53(\mathrm{~m}, 1 \mathrm{H}), 2.46-2.38(\mathrm{~m}, 1 \mathrm{H})$, 1.68 (brs, $2 \mathrm{H}) ;{ }^{13} \mathrm{C} \mathrm{NMR}\left(75 \mathrm{MHz}, \mathrm{CDCl}_{3}\right) \delta 150.7,134.8,126.5,123.6,122.7,118.2,51.3$, 44.7; HR-ESIMS calcd for $\mathrm{C}_{8} \mathrm{H}_{12} \mathrm{NS}\left(\mathrm{M}+\mathrm{H}^{+}\right)$154.0690, found 154.0687. 
(E)-1-Phenylhexa-1,5-dien-3-amine (3h): ${ }^{10}$ 75\% (Method A); ${ }^{1} \mathrm{H}$ NMR (300 $\mathrm{MHz}, \mathrm{CDCl}_{3}$ )

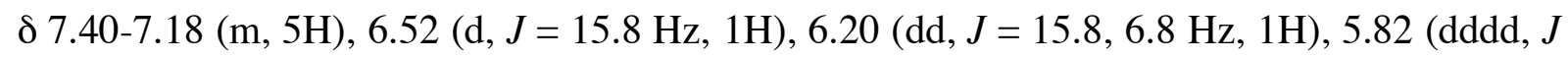
$=17.0,10.2,7.7,6.6 \mathrm{~Hz}, 1 \mathrm{H}), 5.19-5.09(\mathrm{~m}, 2 \mathrm{H}), 3.58(\mathrm{dddd}, J=7.3,6.8,5.7,1.1 \mathrm{~Hz}, 1 \mathrm{H})$, 2.36 (dddt, $J=13.7,6.6,5.7,1.3 \mathrm{~Hz}, 1 \mathrm{H}), 2.24$ (dddt, $J=13.7,7.7,7.3,1.1 \mathrm{~Hz}, 1 \mathrm{H}), 1.48$ (brs, $2 \mathrm{H}) ;{ }^{13} \mathrm{C}$ NMR $\left(75 \mathrm{MHz}, \mathrm{CDCl}_{3}\right) \delta 137.1,135.0,134.2,129.0,128.6,127.3,126.3,117.8$, 53.2, 42.5; HR-ESIMS calcd for $\mathrm{C}_{12} \mathrm{H}_{16} \mathrm{~N}\left(\mathrm{M}+\mathrm{H}^{+}\right)$174.1283, found 174.1284.

1-Phenylhex-5-en-3-amine (3i): ${ }^{11}$ 78\% (Method C); ${ }^{1} \mathrm{H}$ NMR $\left(300 \mathrm{MHz}, \mathrm{CDCl}_{3}\right) \delta$ 7.32-7.24 (m, 2H), 7.22-7.14 (m, 3H), 5.79 (dddd, $J=17.6,9.6,8.0,6.4 \mathrm{~Hz}, 1 \mathrm{H}), 5.12-5.06(\mathrm{~m}, 2 \mathrm{H})$, $2.83(\mathrm{tt}, J=8.0,4.9 \mathrm{~Hz}, 1 \mathrm{H}), 2.76(\mathrm{ddd}, J=13.7,10.0,5.9 \mathrm{~Hz}, 1 \mathrm{H}), 2.64(\mathrm{ddd}, J=13.7,10.0$, $6.4 \mathrm{~Hz}, 1 \mathrm{H}$ ), 2.27 (dddt, $J=13.7,6.4,4.7,1.4 \mathrm{~Hz}, 1 \mathrm{H}$ ), 2.03 (dddt, $J=13.7,7.9,7.9,1.0 \mathrm{~Hz}$, $1 \mathrm{H}), 1.76$ (dddd, $J=13.5,10.0,6.4,4.9 \mathrm{~Hz}, 1 \mathrm{H}), 1.61$ (dddd, $J=13.5,10.0,7.9,5.9 \mathrm{~Hz}, 1 \mathrm{H})$, 1.26 (brs, 2H); ${ }^{13} \mathrm{C} \mathrm{NMR}\left(75 \mathrm{MHz}, \mathrm{CDCl}_{3}\right) \delta 142.2$, 135.6, 128.36, 128.34, 125.8, 117.4, 50.2, 42.7, 39.4, 32.6; HR-ESIMS calcd for $\mathrm{C}_{12} \mathrm{H}_{18} \mathrm{~N}\left(\mathrm{M}+\mathrm{H}^{+}\right)$176.1439, found 176.1435.

1-Cyclohexylbut-3-en-1-amine (3j): 80\% (Method C); ${ }^{1} \mathrm{H}$ NMR (300 MHz, $\left.\mathrm{CDCl}_{3}\right) \delta 5.80$ (dddd, $J=16.9,10.4,8.3,6.1 \mathrm{~Hz}, 1 \mathrm{H}), 5.13-5.05$ (m, 2H), 2.57 (ddd, $J=9.0,5.3,3.9 \mathrm{~Hz}, 1 \mathrm{H}$ ), 2.34-2.24 (m, 1H), 1.96 (apparent ddd, $J=13.7,9.0,8.3 \mathrm{~Hz}, 1 \mathrm{H}), 1.82-1.61(\mathrm{~m}, 5 \mathrm{H}), 1.32-$ 0.93 (m, 8H); $\left.{ }^{13} \mathrm{C} \mathrm{NMR} \mathrm{(100} \mathrm{MHz,} \mathrm{CDCl}_{3}\right) \delta$ 136.6, 117.0, 55.3, 43.4, 39.4, 29.7, 28.3, 26.6, 26.5, 26.4; HR-ESIMS calcd for $\mathrm{C}_{10} \mathrm{H}_{20} \mathrm{~N}\left(\mathrm{M}+\mathrm{H}^{+}\right)$154.1596, found 154.1593.

2-Phenylhex-5-en-3-amine (3k): 69\% (syn/anti = 73/27) (Method C); ${ }^{1} \mathrm{H}$ NMR (400 MHz, $\mathrm{CDCl}_{3}$ ) syn-isomer $\delta 7.34-7.15(\mathrm{~m}, 5 \mathrm{H}), 5.75$ (dddd, $\left.J=17.8,9.4,8.3,6.0 \mathrm{~Hz}, 1 \mathrm{H}\right), 5.08-5.02$ $(\mathrm{m}, 2 \mathrm{H}), 2.93(\mathrm{ddd}, J=9.0,6.8,3.9 \mathrm{~Hz}, 1 \mathrm{H}), 2.69(\mathrm{dq}, J=6.8,7.1 \mathrm{~Hz}, 1 \mathrm{H}), 2.16$ (dddt $J=$ 13.8, 6.0, 3.9, $1.6 \mathrm{~Hz}, 1 \mathrm{H}), 1.86$ (dddt, $J=13.8,9.0,8.3,1.0 \mathrm{~Hz}, 1 \mathrm{H}), 1.31(\mathrm{~d}, J=7.1 \mathrm{~Hz}, 3 \mathrm{H})$, 1.20 (brs, 2H); anti-isomer (representative peaks) $\delta 5.84$ (dddd, $J=17.1,10.2,8.5,6.1 \mathrm{~Hz}$, 5H), 5.17-5.09 (m, 2H), 2.64 (apparent quint, $J=7.2 \mathrm{~Hz}, 1 \mathrm{H}), 2.44(\mathrm{~m}, 1 \mathrm{H}), 1.86$ (dddt, $J=$ 13.9, 9.0, 8.3, $1.0 \mathrm{~Hz}, 1 \mathrm{H}), 1.28(\mathrm{~d}, J=7.1 \mathrm{~Hz}, 3 \mathrm{H}) ;{ }^{13} \mathrm{C} \mathrm{NMR}\left(100 \mathrm{MHz}, \mathrm{CDCl}_{3}\right)$ synisomer $\delta 145.2,136.2,128.3,127.7,126.2,117.3,56.0,45.6,40.0,16.4$; HR-ESIMS calcd for $\mathrm{C}_{12} \mathrm{H}_{18} \mathrm{~N}\left(\mathrm{M}+\mathrm{H}^{+}\right)$176.1439, found 176.1444.

Assignment of the relative configuration of 3k: A mixture of $3 \mathbf{k}(20.6 \mathrm{mg})$, propionyl chloride (15 $\mu \mathrm{L}, 1.5$ equiv) and triethylamine (2 equiv) in dichloromethane ( $2 \mathrm{~mL})$ was stirred at $\mathrm{rt}$ for $5 \mathrm{~h}$. The resultant was purified by preparative TLC (hexane/ethyl acetate $=1 / 1$ ) to give the corresponding propionamide $(26.2 \mathrm{mg})$. Then, a mixture of propionamide $(24.3 \mathrm{mg})$ and $\mathrm{Pd} / \mathrm{C}(5 \mathrm{wt} \%$, wet) in ethanol $(2 \mathrm{~mL})$ was stirred at $\mathrm{rt}$ for $29 \mathrm{~h}$ under hydrogen atmosphere 
using a balloon (ca. $1 \mathrm{~atm}$ ). The mixture was filtered through a Celite pad and concentrated in vacuo to dryness. The crude hydrogenated product was then treated with lithium aluminum hydride (10.3 mg, 2 equiv) in THF $(2 \mathrm{~mL})$ at $80{ }^{\circ} \mathrm{C}$ for $6 \mathrm{~h}$. The mixture was cooled to $\mathrm{rt}$ and quenched by a careful addition of powdered $\mathrm{Na}_{2} \mathrm{SO}_{4} \bullet 10 \mathrm{H}_{2} \mathrm{O}(89.5 \mathrm{mg})$. After being mixed until colorless precipitates were formed, the mixture was filtered through a Celite pad, concentrated in vacuo and purified by preparative TLC (hexane/isopropylamine $=10 / 1$ ) to give 2-phenyl- $N$-propylhexan-3-amine $(12.3 \mathrm{mg}) .{ }^{1} \mathrm{H}$ NMR analysis of this amine (10\% $\mathrm{CDCl}_{3}$ in $\left.\mathrm{CCl}_{4}\right)$ showed two sets of doublets [ $\delta 1.27(\mathrm{~J}=7.1 \mathrm{~Hz})$ (major), $1.23(\mathrm{~J}=7.1 \mathrm{~Hz})$ (minor)]. According to the literature, ${ }^{12}$ this indicates that the major diastereomer is syn isomer. 2-Aminopent-4-enoic acid (3l): ${ }^{13}$ Quant. (Method B); decomp. 247-248 ${ }^{\circ} \mathrm{C} ;{ }^{1} \mathrm{H}$ NMR (300 $\mathrm{MHz}, \mathrm{D}_{2} \mathrm{O}, 1,4$-dioxane as an internal standard: $\left.\delta 3.75 \mathrm{ppm}\right) \delta 5.77$ (ddt, $J=17.2,10.0,7.3$ $\mathrm{Hz}, 1 \mathrm{H}), 5.27$ (brd, $J=17.2 \mathrm{~Hz}, 1 \mathrm{H}), 5.26$ (brd, $J=10.0 \mathrm{~Hz}, 1 \mathrm{H}), 3.80$ (brt, $J=6.0 \mathrm{~Hz}, 1 \mathrm{H})$, 2.66 (br ddd, $J=14.7,7.3,6.0 \mathrm{~Hz}, 1 \mathrm{H}), 2.59$ (br ddd, $J=14.7,7.3,6.0 \mathrm{~Hz}, 1 \mathrm{H}) ;{ }^{13} \mathrm{C}$ NMR $(75$ $\mathrm{MHz}, \mathrm{D}_{2} \mathrm{O}, 1,4$-dioxane as an internal standard: $\left.\delta 67.19 \mathrm{ppm}\right) \delta 174.7,131.9,121.1,54.6$, 35.4, 20.9. Anal. Calcd for $\mathrm{C}_{5} \mathrm{H}_{9} \mathrm{NO}_{2}$ : C, 52.16; H, 7.88; N, 12.17. Found: C, 51.88; H, 7.72; N, 11.97 .

\section{Diastereoselective $\alpha$-aminoallylation of $(S)$-1m (Scheme 2)}

Allylboronate $2(106.0 \mathrm{mg}, 0.6 \mathrm{mmol})$ in ethanol $(0.5 \mathrm{~mL})$ was added to a mixture of liquid ammonia (ca. $0.5 \mathrm{~mL}$ ) and ethanol $(0.5 \mathrm{~mL})$ at $-78{ }^{\circ} \mathrm{C}$ (see Method A). After being stirred at $0{ }^{\circ} \mathrm{C}$ for $30 \mathrm{~min}$, aldehyde $(S)-1 \mathrm{~m}(132.5 \mathrm{mg}, 0.5 \mathrm{mmol},>94 \%$ ee $)$ in ethanol $(0.5$ $\mathrm{mL}$ ) was added to the suspension. The mixture was stirred at $0{ }^{\circ} \mathrm{C}$ for $12 \mathrm{~h}$ and concentrated in vacuo. The crude was purified by preparative TLC (hexane/isopropylamine $=100 / 7$ ) to give amine 3m (102.6 mg, 67\%, syn/anti = 85/15) as colorless oil.

(2S)-2-(tert-Butyldimethylsilyloxy)-1-phenylhex-5-en-3-amine (3m): $[\alpha]^{26}-12.7$ (c 1.05, $\mathrm{MeOH}, 95 \%$ ee, syn/anti $=85 / 15) ;{ }^{1} \mathrm{H} \mathrm{NMR}\left(400 \mathrm{MHz}, \mathrm{CDCl}_{3}\right)$ syn-isomer $\delta 7.31-7.15(\mathrm{~m}$, $5 \mathrm{H}), 5.72$ (dddd, $J=15.3,11.9,7.8,6.1 \mathrm{~Hz}, 1 \mathrm{H}), 5.08-5.00$ (m, 2H), 3.80 (apparent dt, $J=2.7$, $6.8 \mathrm{~Hz}, 1 \mathrm{H}), 2.97(\mathrm{dd}, J=13.4,7.1 \mathrm{~Hz}, 1 \mathrm{H}), 2.71(\mathrm{dd}, J=13.4,6.4 \mathrm{~Hz}, 1 \mathrm{H}), 2.71(\mathrm{dd}, J=$ 13.4, 6.4 Hz, 1H), 2.62 (ddd, $J=9.0,4.4,2.7 \mathrm{~Hz}, 1 \mathrm{H}$ ), 2.27 (dddt, $J=13.9,6.1,4.4,1.4 \mathrm{~Hz}$, 1H), 2.01 (apparent dt, $J=13.9,8.5 \mathrm{~Hz}, 1 \mathrm{H}), 1.31$ (brs, $2 \mathrm{H}), 0.89$ (s, 9H), 0.02 (s, 3H), -0.15 
(s, 3H); anti-isomer (representative peaks) $\delta 5.82$ (dddd, $J=17.2,10.0,7.1,7.0 \mathrm{~Hz}, 1 \mathrm{H}$ ), 5.18-5.08 (m, 2H), 2.87 (ddd, $J=8.8,5.2,3.5 \mathrm{~Hz}, 1 \mathrm{H}$ ), 2.12 (apparent dt, $J=13.9,8.2 \mathrm{~Hz}$, $1 \mathrm{H}), 0.83(\mathrm{~s}, 9 \mathrm{H}),-0.09(\mathrm{~s}, 3 \mathrm{H}),-0.40(\mathrm{~s}, 3 \mathrm{H}) ;{ }^{13} \mathrm{C} \mathrm{NMR}\left(100 \mathrm{MHz}, \mathrm{CDCl}_{3}\right)$ syn-isomer $\delta$ 138.9, 136.4, 129.6, 128.3, 126.1, 116.9, 76.5, 53.1, 40.2, 39.5, 25.90, 18.08, -4.6; anti-isomer ठ 139.3, 136.0, 129.8, 128.1, 126.0, 117.2, 77.1, 55.0, 37.74, 37.71, 25.84, 17.98, -4.8, -5.3;

HR-ESIMS calcd for $\mathrm{C}_{18} \mathrm{H}_{32} \mathrm{NOSi}\left(\mathrm{M}+\mathrm{H}^{+}\right)$306.2253, found 306.2259.

\section{Assignment of the stereochemistry:}

(1) (S)-1m was prepared from L-phenylalanine. ${ }^{1}$ The enantiomeric excess of $(S)$-1m was determined by HPLC analysis to be $94 \%$ ee after its conversion to the corresponding primary alcohol by $\mathrm{NaBH}_{4}$-reduction. HPLC (CHIRALCEL OD-H, $0.46 \mathrm{~cm} \varnothing \times 25 \mathrm{cmL}$, hexane $/ 2$-propanol $=9 / 1$, flow rate $1.0 \mathrm{~mL} / \mathrm{min}$, UV detection at $254 \mathrm{~nm}) t_{R}=4.1 \mathrm{~min}(S)$, $9.5 \min (R)$.

(2) The authentic racemic product $( \pm)-\mathbf{3 m}$ was prepared from $( \pm)-\mathbf{1 m}$ in $72 \%$ (syn/anti $=$ $85 / 15)$ according to the above procedure. By HPLC analysis using $( \pm)-3 m$, the enantiomeric excess of (2S)-3m was determined to be $95 \%$ ee. HPLC (double CHIRALCEL OD columns, $0.46 \mathrm{~cm} \varnothing \times 25 \mathrm{cmL}$ each, hexane/2-propanol = 9/1, flow rate $0.3 \mathrm{~mL} / \mathrm{min}$, UV detection at $254 \mathrm{~nm}) t_{R}=26.3 \mathrm{~min}(2 S, 3 S), 27.6 \mathrm{~min}(2 S, 3 R), 32.4 \mathrm{~min}$ $(2 R, 3 S), 61.7 \min (2 R, 3 R)$.

(3) The relative configuration of $\mathbf{3 m}$ was determined as follows (Table S-1). To a solution of $( \pm)-3 \mathrm{~m}(72.2 \mathrm{mg}, \mathrm{dr}=85 / 15)$ in dry THF $(2 \mathrm{~mL})$ was added TBAF (1.0 M THF solution, $354 \mu \mathrm{L})$ at $\mathrm{rt}$. The mixture was stirred at $\mathrm{rt}$ for $45 \mathrm{~h}$, diluted with ethyl acetate, washed with sat. aq. $\mathrm{NaHCO}_{3}$ and brine, dried over anhydrous $\mathrm{MgSO}_{4}$, filtered and concentrated to dryness. The residue was dissolved in dry THF $(1.0 \mathrm{~mL})$ and treated with 1,1 'carbonyldiimidazole $(42 \mathrm{mg})$ in THF $(0.5 \mathrm{~mL})$ at $\mathrm{rt}$. The mixture was stirred at $\mathrm{rt}$ for $4 \mathrm{~h}$, concentrated and purified preparative TLC (hexane/ethyl acetate 1/1) to give oxazolidinone 8 (28.9 mg, 2 steps $56 \%$, trans/cis $=85 / 15)$. NOE difference spectroscopy with $\mathbf{8}$ determined the relative configuration of the major diastereomer to be trans.

4-Allyl-5-benzyloxazolidin-2-one (8): ${ }^{1} \mathrm{H} \mathrm{NMR}\left(400 \mathrm{MHz}, \mathrm{CDCl}_{3}\right)$ trans-isomer $\delta$ 7.35-7.19 (m, 5H; Ph), 6.28 (brs, 1H; NH), 5.58 (ddt, $J=17.1,10.3,7.1 \mathrm{~Hz}, 1 \mathrm{H} ; \mathrm{H} 5), 5.14-5.02$ (m, 2H, H6), 4.44 (dt, $J=5.6,6.5 \mathrm{~Hz}, 1 \mathrm{H} ; \mathrm{H} 2$ ), 3.59 (dt, $J=5.6,6.4 \mathrm{~Hz}, 1 \mathrm{H} ; \mathrm{H} 3$ ), 3.09 (dd, $J=14.0$, $\left.6.5 \mathrm{~Hz}, 1 \mathrm{H} ; \mathrm{H} 1_{\mathrm{a}}\right), 2.91(\mathrm{dd}, J=14.0,6.5 \mathrm{~Hz}, 1 \mathrm{H} ; \mathrm{H} 1 \mathrm{~b}), 2.16(\mathrm{dt}, J=14.4,6.4 \mathrm{~Hz}, 1 \mathrm{H} ; \mathrm{H} 4)$, $2.13(\mathrm{dt}, J=14.4,6.4 \mathrm{~Hz}, 1 \mathrm{H}$; H4); cis-isomer (representative peaks) $\delta 6.15$ (brs, 1H), 5.75 (dddd, $J=16.7,10.5,7.7,6.0 \mathrm{~Hz}, 1 \mathrm{H}), 5.24-5.17(\mathrm{~m}, 2 \mathrm{H}), 4.88(\mathrm{ddd}, J=9.6,7.6,4.6 \mathrm{~Hz}, 1 \mathrm{H})$, 
$3.86(\mathrm{ddd}, J=9.6,7.4,4.0 \mathrm{~Hz}, 1 \mathrm{H}), 2.45-2.37(\mathrm{~m}, 1 \mathrm{H}), 2.35-2.25(\mathrm{~m}, 1 \mathrm{H}) ;{ }^{13} \mathrm{C} \mathrm{NMR}(100$ $\mathrm{MHz}, \mathrm{CDCl}_{3}$ ) trans-isomer $\delta 159.0,135.2,131.9,129.4,128.6,127.1,119.3,81.7,56.1,40.4$, 39.3; cis-isomer $\delta 159.1,136.4,132.7,129.0,126.9,119.5,80.1,54.7,35.5,34.7$; HR-ESIMS calcd for $\mathrm{C}_{13} \mathrm{H}_{16} \mathrm{NO}_{2}\left(\mathrm{M}+\mathrm{H}^{+}\right)$218.1181, found 218.1183.

Table S-1. NOE Difference Spectroscopy with 8

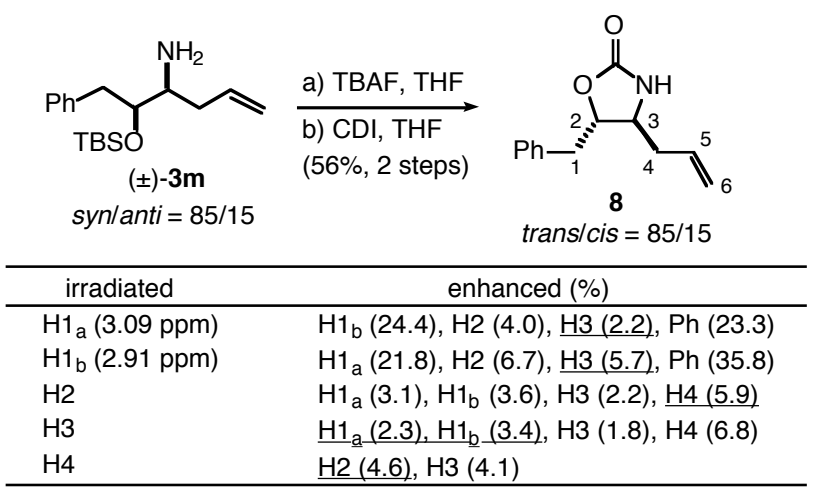

\section{Diastereoselective $\alpha$-aminoallylation of $(2 S, 3 S)$-1n (Scheme 2)}

$(2 S, 3 S)-1 n$ was prepared from L-chinovose and used without further purification according to the literature. ${ }^{2}$ A solution of crude $(2 S, 3 S)-\mathbf{1 n}(67.2 \mathrm{mg}$, obtained from $164 \mathrm{mg}$ of L-chinovose) in liquid ammonia (ca. $0.5 \mathrm{~mL})$ and ethanol $(1 \mathrm{~mL})$ was stirred at $0{ }^{\circ} \mathrm{C}$ for $1 \mathrm{~h}$ (see Method A). Allylboronate $2(84 \mathrm{mg}, 0.5 \mathrm{mmol})$ in ethanol $(0.5 \mathrm{~mL})$ was added to the mixture. After being stirred at $0{ }^{\circ} \mathrm{C}$ for $24 \mathrm{~h}$, the cloudy solution was collected with methanol and evaporated. The residue was charged on a DOWEX 50W-X2 column (ca. $5 \mathrm{~g} /$ wet, pretreated with $1 \mathrm{~N}$ aq. $\mathrm{HCl}$, water and methanol) with methanol, washed with methanol (ca. 100 $\mathrm{mL}$ ) and eluted with $0.2 \mathrm{~N}$ ammonia in methanol. The fractions were checked by TLC plates without development using a phosphomolybdic acid indicator. Positive fractions were concentrated to dryness and the residue was dissolved in dichloromethane $(1 \mathrm{~mL})$ and treated with $\mathrm{Boc}_{2} \mathrm{O}(125 \mu \mathrm{L})$ at $\mathrm{rt}$ for $4 \mathrm{~h}$. The mixture was evaporated and purified by flash silica gel column chromatography to give Boc-3n $(62.2 \mathrm{mg}, 51 \%$ based on $\mathbf{2},>98 \%$ syn, the minor isomer was not detected by ${ }^{1} \mathrm{H}$ NMR analysis).

$N$-tert-Butoxycarbonyl-4-aminohept-6-ene-2,3-diol (Boc-3n): $[\alpha]_{\mathrm{D}}^{27}-8.7$ (c $0.52, \mathrm{MeOH}$, $>98 \%$ syn); ${ }^{1} \mathrm{H}$ NMR (300 MHz, $\mathrm{CDCl}_{3}$ ) $\delta 5.79$ (ddt, $\left.J=17.0,10.1,7.0 \mathrm{~Hz}, 1 \mathrm{H}\right), 5.17-5.04$ (m, 2H), 4.75 (brd, $J=7.7 \mathrm{~Hz}, 1 \mathrm{H}), 3.97$ (apparent q, $J=7.2 \mathrm{~Hz}, 1 \mathrm{H}), 3.49$ (dq, $J=8.3,6.2 \mathrm{~Hz}$, $1 \mathrm{H}), 3.26$ (dd, $J=8.3,1.4 \mathrm{~Hz}, 1 \mathrm{H}), 3.90$ (brs, 2H), 2.42-2.23 (m, 2H), 1.43 (s, 9H), 1.27 (d, $J$ 
$=6.2 \mathrm{~Hz}, 3 \mathrm{H}) ;{ }^{13} \mathrm{C} \mathrm{NMR}\left(75 \mathrm{MHz}, \mathrm{CDCl}_{3}\right) \delta 157.5,134.5,117.7,80.3,77.4,67.5,50.2,36.6$,

28.3, 19.2; HR-ESIMS calcd for $\mathrm{C}_{12} \mathrm{H}_{24} \mathrm{NO}_{4}\left(\mathrm{M}+\mathrm{H}^{+}\right)$246.1705, found 246.1711.

\section{Transformation of Boc-3n to a L-acosamine derivative (Scheme 2)}

Ozone/oxygen gas was bubbled into a solution of Boc-3n (63 mg, $0.26 \mathrm{mmol})$ in methanol $(4 \mathrm{~mL})$ at $-78{ }^{\circ} \mathrm{C}$ until the solution turned purple. After removal of excess ozone, dimethyl sulfide $(97 \mu \mathrm{L})$ was added dropwise to the solution at $-78{ }^{\circ} \mathrm{C}$. The mixture was wormed to $\mathrm{rt}$ and stirred at $\mathrm{rt}$ for $2 \mathrm{~h}$. The mixture was recovered with methanol and concentrated to dryness. The residue was dissolved in dry methanol $(2.5 \mathrm{~mL})$ and treated with sat. $\mathrm{HCl} / \mathrm{MeOH}$ (ca. $0.5 \mathrm{~mL}$ ). After being stirred at $\mathrm{rt}$ for $6 \mathrm{~h}$, the mixture was concentrated in vacuo. The residue was then treated with acetic anhydride $(194 \mu \mathrm{L})$ in pyridine $(1 \mathrm{~mL})$ at $\mathrm{rt}$ for $9 \mathrm{~h}$. After removal of pyridine under reduced pressure at $50{ }^{\circ} \mathrm{C}$, the residue was purified by preparative TLC (ethyl acetate) to give 1-O-methyl-3- $\mathrm{N}$-acetyl-4-Oacetyl-L-acosamine (27.7 mg, 3 steps $44 \%$ ) as colourless solid. Recrystallization from dichloromethane/hexane gave colourless needles (18.1 mg, $\alpha$-anomer).

1-O-Methyl-3- $\boldsymbol{N}$-acetyl-4- $\boldsymbol{O}$-acetyl-L-acosamine: ${ }^{14} \underline{\alpha \text {-anomer }}$ mp $159-161{ }^{\circ} \mathrm{C},[\alpha]^{26}{ }_{\mathrm{D}}-190.3$ (c 0.38, MeOH); $\left.{ }^{1} \mathrm{H} \mathrm{NMR} \mathrm{(400} \mathrm{MHz,} \mathrm{CDCl}_{3}\right) \delta 5.57$ (brd, $\left.J=8.3 \mathrm{~Hz}, 1 \mathrm{H}\right), 4.70(\mathrm{dd}, J=10.2$, $8.8 \mathrm{~Hz}, 1 \mathrm{H}), 4.46(\mathrm{dd}, J=10.2,8.8 \mathrm{~Hz}, 1 \mathrm{H}), 4.40(\mathrm{dddd}, J=11.8,10.2,8.3,4.5 \mathrm{~Hz}, 1 \mathrm{H}), 3.89$ (dq, $J=8.8,6.4 \mathrm{~Hz}, 1 \mathrm{H}), 3.33$ (s, 3H), 2.21 (ddd, $J=13.2,4.5,1.2 \mathrm{~Hz}, 1 \mathrm{H}), 2.07$ (s, 3H), 1.90 $(\mathrm{s}, 3 \mathrm{H}), 1.58(\mathrm{ddd}, J=13.2,11.8,3.5 \mathrm{~Hz}, 3 \mathrm{H}), 1.17(\mathrm{~d}, J=6.4 \mathrm{~Hz}, 3 \mathrm{H}) ;{ }^{13} \mathrm{C} \mathrm{NMR}(100 \mathrm{MHz}$, $\left.\mathrm{CDCl}_{3}\right) \delta 171.8,169.7,97.5,75.7,65.6,54.6,47.0,36.4,23.4,20.9$, 17.7; HR-ESIMS calcd for $\mathrm{C}_{11} \mathrm{H}_{20} \mathrm{NO}_{5}\left(\mathrm{M}+\mathrm{H}^{+}\right)$246.1341, found 246.1342.

\section{$\alpha$-Aminocrotylation of aromatic aldehydes (Scheme 3)}

$(E)$ - or $(Z)$-Crotylboronate $5(0.75 \mathrm{mmol})$ in ethanol $(0.5 \mathrm{~mL})$ was added to liquid ammonia (ca. $0.5 \mathrm{~mL}$, see Method A) at $-78{ }^{\circ} \mathrm{C}$ and rinsed with ethanol $(0.5 \mathrm{~mL})$. Colorless precipitates were formed during addition of $\mathbf{5}$. After being stirred at $\mathrm{rt}$ for $30 \mathrm{~min}$, aldehyde $1 \mathbf{a}(0.5 \mathrm{mmol})$ or a 1,4 -dioxane solution of aldehyde $\mathbf{1 b}(0.5 \mathrm{mmol}$ in $0.5 \mathrm{~mL}$ of the solvent $)$ was added to the suspension and rinsed with ethanol $(0.5 \mathrm{~mL})$ or 1,4-dioxane $(0.5 \mathrm{~mL})$, respectively. The mixture was stirred at $\mathrm{rt}$ for $2 \mathrm{~h}$ and worked up in the same way as Method A. 
$\left(1 S^{*}, 2 R^{*}\right)$-2-Methyl-1-phenylbut-3-en-1-amine $\left(\right.$ syn-6a): ${ }^{15} 85 \% \quad($ syn/anti $=>99 /<1) ;{ }^{1} \mathrm{H}$ NMR (400 MHz, $\left.\mathrm{CDCl}_{3}\right) \delta$ 7.35-7.19 (m, 5H), 5.69 (ddd, $\left.J=17.8,9.7,7.3 \mathrm{~Hz}, 1 \mathrm{H}\right), 5.06-$ $4.97(\mathrm{~m}, 2 \mathrm{H}), 3.88(\mathrm{~d}, J=5.5 \mathrm{~Hz}, 1 \mathrm{H}), 2.50$ (ddq, $J=7.3,5.5,6.8 \mathrm{~Hz}, 1 \mathrm{H}), 1.52$ (brs, 2H), $0.98(\mathrm{~d}, J=6.8 \mathrm{~Hz}, 3 \mathrm{H}) ;{ }^{13} \mathrm{C} \mathrm{NMR}\left(100 \mathrm{MHz}, \mathrm{CDCl}_{3}\right) \delta 144.3,141.0,128.0,127.1,126.8$, 115.0, 60.0, 44.7, 15.0; HR-ESIMS calcd for $\mathrm{C}_{11} \mathrm{H}_{16} \mathrm{~N}\left(\mathrm{M}+\mathrm{H}^{+}\right)$162.1283, found 162.1279.

$\left(1 S^{*}, 2 S^{*}\right)$-2-Methyl-1-phenylbut-3-en-1-amine $\left(\right.$ anti-6a) $:{ }^{15} 79 \%($ syn/anti $=7 / 93) ;{ }^{1} \mathrm{H}$ NMR $\left(400 \mathrm{MHz}, \mathrm{CDCl}_{3}\right) \delta$ 7.34-7.22 (m, 5H), 5.75 (ddd, $\left.J=17.1,10.3,8.5 \mathrm{~Hz}, 1 \mathrm{H}\right), 5.17$ (dq, $J=$ 17.1, 1.0 Hz, 1H), $5.12(\mathrm{dd}, J=10.3,1.7 \mathrm{~Hz}, 1 \mathrm{H}), 3.65(\mathrm{~d}, J=8.5 \mathrm{~Hz}, 1 \mathrm{H}), 2.38(\mathrm{ddq}, J=8.5$, 8.5, $6.8 \mathrm{~Hz}, 1 \mathrm{H}), 1.75$ (brs, 2H), 0.82 (d, $J=6.8 \mathrm{~Hz}, 3 \mathrm{H}) ;{ }^{13} \mathrm{C} \mathrm{NMR}\left(100 \mathrm{MHz}, \mathrm{CDCl}_{3}\right) \delta$ 144.6, 141.7, 128.3, 127.3, 127.1, 115.8, 60.7, 46.3, 17.7; HR-ESIMS calcd for $\mathrm{C}_{11} \mathrm{H}_{16} \mathrm{~N}$ $\left(\mathrm{M}+\mathrm{H}^{+}\right) 162.1283$, found 162.1278 .

$\left(1 S^{*}, 2 R^{*}\right)$-2-Methyl-1-(4-nitrophenyl)but-3-en-1-amine $\quad($ syn-6b): $90 \% \quad($ syn/anti $=$ $>99 /<1$ ); ${ }^{1} \mathrm{H}$ NMR (300 MHz, $\mathrm{CDCl}_{3}$ ) $\delta 8.18$ (apparent d, $J=8.3 \mathrm{~Hz}, 2 \mathrm{H}$ ), 7.47 (apparent d, $J$ $=8.3 \mathrm{~Hz}, 2 \mathrm{H}), 5.66(\mathrm{ddd}, J=17.0,10.6,7.3 \mathrm{~Hz}, 1 \mathrm{H}), 5.05$ (apparent d, $J=10.6 \mathrm{~Hz}, 1 \mathrm{H}), 5.03$ (apparent d, $J=17.0 \mathrm{~Hz}, 1 \mathrm{H}), 4.03(\mathrm{~d}, J=5.5 \mathrm{~Hz}, 1 \mathrm{H}), 2.52(\mathrm{ddq}, J=7.3,5.5,6.8 \mathrm{~Hz}, 1 \mathrm{H})$, 1.55 (brs. 2H), 0.98 (d, $J=6.8 \mathrm{~Hz}, 3 \mathrm{H}) ;{ }^{13} \mathrm{C} \mathrm{NMR}\left(100 \mathrm{MHz}, \mathrm{CDCl}_{3}\right.$ ) $\delta$ 152.0, 146.9, 140.0, 128.0, 123.2, 115.9, 59.5, 44.7, 14.6; HR-ESIMS calcd for $\mathrm{C}_{11} \mathrm{H}_{15} \mathrm{~N}_{2} \mathrm{O}_{2}\left(\mathrm{M}+\mathrm{H}^{+}\right)$207.1134, found 207.1136.

$\left(1 S^{*}, 2 S^{*}\right)$-2-Methyl-1-(4-nitrophenyl)but-3-en-1-amine (anti-6b): $92 \%$ (syn/anti = 2/98); mp 43-44 ${ }^{\circ} \mathrm{C} ;{ }^{1} \mathrm{H}$ NMR (400 MHz, $\left.\mathrm{CDCl}_{3}\right) \delta 8.19$ (apparent d, $J=8.8 \mathrm{~Hz}, 2 \mathrm{H}$ ), 7.51 (apparent d, $J=8.8 \mathrm{~Hz}, 2 \mathrm{H}), 5.69$ (ddd, $J=17.1,10.2,8.6 \mathrm{~Hz}, 1 \mathrm{H}), 5.16$ (apparent d, $J=17.1 \mathrm{~Hz}, 1 \mathrm{H}$ ), 5.15 (apparent d, $J=10.2 \mathrm{~Hz}, 1 \mathrm{H}), 3.82(\mathrm{~d}, J=8.1 \mathrm{~Hz}, 1 \mathrm{H}), 2.38(\mathrm{ddq}, J=8.6,8.1,6.8 \mathrm{~Hz}$, 1H), 1.89 (brs. $2 \mathrm{H}), 0.85$ (d, $J=6.8 \mathrm{~Hz}, 3 \mathrm{H}) ;{ }^{13} \mathrm{C} \mathrm{NMR}\left(75 \mathrm{MHz}, \mathrm{CDCl}_{3}\right) \delta 152.2,147.1$, $140.3,128.2$, 123.4, 116.7, 60.1, 46.1, 17.3; HR-EIMS calcd for $\mathrm{C}_{11} \mathrm{H}_{15} \mathrm{~N}_{2} \mathrm{O}_{2}\left(\mathrm{M}+\mathrm{H}^{+}\right)$ 207.1134, found 207.1142.

\section{Synthesis of alloisoleucine (Scheme 4)}

(Z)-Crotylboronate $5(137.5 \mathrm{mg}, 0.75 \mathrm{mmol})$ in ethanol $(0.5 \mathrm{~mL})$ was added to liquid ammonia (ca. $0.5 \mathrm{~mL}$, see Method A) at $-78{ }^{\circ} \mathrm{C}$ and rinsed with ethanol $(0.5 \mathrm{~mL})$. After being stirred at $-10^{\circ} \mathrm{C}$ for $30 \mathrm{~min}$, glyoxylic acid (11) $(46.5 \mathrm{mg}, 0.5 \mathrm{mmol})$ in water $(0.5 \mathrm{~mL})$ was added to the suspension and rinsed with ethanol $(0.5 \mathrm{~mL})$. The mixture was stirred at $-10{ }^{\circ} \mathrm{C}$ for $3 \mathrm{~h}$. Most of the ammonia was removed by aspiration. After addition of $\mathrm{Pd} / \mathrm{C}(5$ 
$\mathrm{wt} \%$, wet, $15.2 \mathrm{mg}$ ), the mixture was stirred at $\mathrm{rt}$ for $12 \mathrm{~h}$ under hydrogen atmosphere using a balloon (ca. $1 \mathrm{~atm}$ ). The mixture was filtered through a Celite pad and concentrated in vacuo. The residue was purified by ion exchange resin chromatography (see the method for isolation of 3l, Method B) to give ( \pm )-alloisoleucine $(60 \mathrm{mg}, 91 \%)$. The above one-pot procedure (subsequent hydrogenation) was important to suppress the undesired isomerization of the syncrotylated product to the linear isomer. This isomerization was observed when we tried to isolate the crotylated product, i. e. concentration with heating.

( \pm )-Alloisoleucine: ${ }^{16}$ Colorless plates (recrystallized from $90 \%$ aqueous ethanol); decomp. $238{ }^{\circ} \mathrm{C} ;{ }^{1} \mathrm{H}$ NMR (400 MHz, $\mathrm{D}_{2} \mathrm{O}, 1,4$-dioxane as an internal standard: $\left.\delta 3.75 \mathrm{ppm}\right) \delta 3.73$ (d, $J=3.6 \mathrm{~Hz}, 1 \mathrm{H}), 2.06(\mathrm{dtq}, J=3.6,7.3,6.6 \mathrm{~Hz}, 1 \mathrm{H}), 1.43(\mathrm{ddt}, J=14.0,7.3,7.6 \mathrm{~Hz}, 1 \mathrm{H}), 1.32$ (ddt, $J=14.0,7.3,7.6 \mathrm{~Hz}, 1 \mathrm{H}), 0.95(\mathrm{t}, J=7.6 \mathrm{~Hz} 3 \mathrm{H}), 0.93(\mathrm{~d}, J=6.6 \mathrm{~Hz}, 3 \mathrm{H}) ;{ }^{13} \mathrm{C} \mathrm{NMR}$ (100 MHz, $\mathrm{D}_{2} \mathrm{O}, 1,4$-dioxane as an internal standard: $\left.\delta 67.19 \mathrm{ppm}\right) \delta 175.2,59.1,36.2,26.1$, 13.9, 11.6; HR-ESIMS calcd for $\mathrm{C}_{6} \mathrm{H}_{14} \mathrm{NO}_{2}\left(\mathrm{M}+\mathrm{H}^{+}\right)$132.1025, found 132.1024.

\section{Enantioselective $\alpha$-aminoallylation of benzaldehyde (Scheme 5)}

A mixture of benzaldehyde $(52.4 \mathrm{mg}, 0.49 \mathrm{mmol})$ in liquid ammonia (ca. $0.5 \mathrm{~mL}$ ) and dichloromethane $\left(1.0 \mathrm{~mL}\right.$ ) was stirred at $-30{ }^{\circ} \mathrm{C}$ for $30 \mathrm{~min}$ (see Method A). Allylboronate $\mathbf{7}^{5}$ $(177.2 \mathrm{mg}, 0.6 \mathrm{mmol})$ in dichloromethane $(0.5 \mathrm{~mL})$ was then added to the mixture and rinsed with dichloromethane $(0.5 \mathrm{~mL})$. The clear solution was stirred at that temperature for $4 \mathrm{~h}$ and worked up in the same way as Method A to give (R)-1-Phenylbut-3-en-1-amine (3a) $(53.4 \mathrm{mg}$, $74 \%$ yield, $34 \%$ ee).

(R)-1-Phenylbut-3-en-1-amine (3a): ${ }^{17}[\alpha]_{\mathrm{D}}^{27}+15.9$ (c 1.30, $\mathrm{CHCl}_{3}, 34 \%$ ee $(R)$ ); HPLC (CHIRALCEL OD-H, $0.46 \mathrm{~cm} \varnothing \times 25 \mathrm{cmL}$, hexane/2-propanol/diethylamine = 96.7/3.3/0.05, flow rate $0.7 \mathrm{~mL} / \mathrm{min}$, UV detection at $254 \mathrm{~nm}) t_{R}=11.0 \mathrm{~min}(R), t_{R}=16.0 \mathrm{~min}(S)$.

\section{Detection of an $N$-unsubstituted imine by ${ }^{1} \mathrm{H}$ NMR analysis}

The following preliminary ${ }^{1} \mathrm{H}$ NMR experiments support involvement of $\mathrm{N}$ unsubstituted imines in the present reaction system. ${ }^{18}$ First, a ${ }^{1} \mathrm{H}$ NMR spectrum of benzaldehyde $(0.2 \mathrm{mmol})$ in ammonia saturated $\mathrm{THF}-\mathrm{d}_{8}(0.6 \mathrm{~mL})$ at $-78{ }^{\circ} \mathrm{C}$ showed two broad doublets at 10.96 and $8.70 \mathrm{ppm}(J=16.0 \mathrm{~Hz})$ and a singlet at $10.00 \mathrm{ppm}$. These doublets could correspond to the $\mathrm{N}$-unsubstituted benzaldimine with a cis geometry of the 
imino hydrogens and the singlet to benzaldehyde. The ratio of imine/aldehyde was $15 / 85$. Next, trimethyl borate $(0.22 \mathrm{mmol})$ was added to this solution at $-78{ }^{\circ} \mathrm{C}$, and the spectrum at that temperature showed a broad doublet at $10.87 \mathrm{ppm}(J=15.8 \mathrm{~Hz}$, corresponding to the imine) and a singlet at $10.00 \mathrm{ppm}$ (benzaldehyde) in a 76/24 ratio. A trace amount of a doublet was also observed at $11.12 \mathrm{ppm}(J=25.4 \mathrm{~Hz})$. These signals were broadened at $-10{ }^{\circ} \mathrm{C}$, but became sharp again when re-cooled to $-78{ }^{\circ} \mathrm{C}$. The coupling partner of the doublet at $10.87 \mathrm{ppm}$ appeared around at $8.7 \mathrm{ppm}$ (confirmed by a decoupling), though the exact value was unclear due to overlapping of unknown signals. Assignment of the imine was further confirmed by similarity of a ${ }^{1} \mathrm{H}$ NMR spectrum of the imine prepared by the Brown's method. ${ }^{19}$ Thus, a spectrum of a mixture of $N$-(trimethylsilyl)benzaldimine $(0.15 \mathrm{mmol})$, trimethyl borate (1.1 equiv), and methanol (1.1 equiv) in $\mathrm{THF}-\mathrm{d}_{8}(0.6 \mathrm{~mL})$ at $-78{ }^{\circ} \mathrm{C}$ showed two doublets at 10.74 and $8.71 \mathrm{ppm}(J=16.0 \mathrm{~Hz})$. The above experiments suggest that $N$ unsubstituted imines are involved under the conditions of $\alpha$-aminoallylation and that conversion of aldehydes to imines appears to be assisted by boron agents. Further investigation concerning the reaction mechanism is now in progress.

\section{References and notes}

(1) Humphrey, A. J.; Turner, N. J.; McCague, R.; Taylor, S. J. C. J. Chem. Soc., Chem. Commun. 1995, 2475 and references therein.

(2) (a) Bilík, V.; Voelter, W.; Bayer, E. Angew. Chem. Int. Ed. 1971, 10, 909. (b) Snyder, J. R.; Serianni, A. S. Carbohydrate Res. 1987, 163, 169.

(3) Roush, W. R.; Walts, A. E. Tetrahedron Lett. 1985, 26, 3427.

(4) (a) Roush, W. R.; Adam, M. A.; Walts, A. E.; Harris, D. J. J. Am. Chem. Soc. 1986, 108, 3422. (b) Hoffmann, R. W.; Ladner, W.; Ditrich, K. Liebigs Ann. Chem. 1989, 883.

(5) Herold, T.; Schrott, U.; Hoffmann, R. W. Chem. Ber. 1981, 114, 359.

(6) van Maarseveen, J. H.; Meester, W. J. N.; Veerman, J. J. N.; Kruse, C. G.; Hermkens, P. H. H.; Rutjes, F. P. J. T.; Hiemstra, H. J. Chem. Soc., Perkin Trans. 1 2001, 994.

(7) Yamada, K.; Hashihayata, T. JP 2002047289, 2002.

(8) (a) Alvaro, G.; Savoia, D. Tetrahedron: Asymmetry 1996, 7, 2083. (b) Aiqiao, M.; Xun, X.; Lanjun, W.; Yaozhong, J. Synth. Commun. 1991, 21, 2207.

(9) Andreoli, P.; Billi, L.; Cainelli, G.; Panunzio, M.; Martelli, G.; Spunta, G. J. Org. Chem. 1990, 55, 4199. 
(10) Hart, D. J.; Kanai, K.; Thomas, D. G.; Yang, T.-K. J. Org. Chem. 1983, 48, 289.

(11) Kobayashi, S.; Ogawa, C.; Konishi, H.; Sugiura, M. J. Am. Chem. Soc. 2003, 125, 6610.

(12) Yamamoto, Y.; Nishii, S.; Maruyama, K.; Komatsu, T.; Ito, W. J. Am. Chem. Soc. 1986, 108,7778 .

(13) Yeh, T.-L.; Liao, C.-C.; Uang, B.-J. Tetrahedron 1997, 53, 1141.

(14) Ginesta, X.; Pastó, M.; Pericas, M. A.; Riera, A. Org. Lett. 2003, 5, 3001.

(15) Hoffmann, R. W.; Endesfelder, A. Liebigs Ann. Chem. 1987, 215.

(16) Lloyd-Williams, P.; Monerris, P.; Gonzalez, I.; Jou, G.; Giralt, E. J. Chem. Soc., Perkin Trans. 1 1994, 1969.

(17) Basile, T.; Bocoum, A.; Savoia, D.; Umani-Ronchi, A. J. Org. Chem. 1994, 59, 7766.

(18) ${ }^{1} \mathrm{H}$ NMR experiments were performed in THF- $\mathrm{d}_{8}$ because assignment of the $N$ unsubstituted imine was difficult when the experiments were carried out in ethanol- $\mathrm{d}_{6}$ probably due to the proton-deuterium exchange. It was confirmed that $\alpha$ aminoallylation of benzaldehyde (1a) with allylboronate 2 in THF (Table 1, Method C) afforded a mixture of amine $3 \mathbf{a}$ and alcohol $4 \mathbf{a}(\mathbf{3 a} / \mathbf{4 a}=55 / 45)$ in quantitative yield (determined by ${ }^{1} \mathrm{H}$ NMR analysis using 1,2,4,5-tetramethylbenzene as an internal standard).

(19) Chen, G.-M.; Brown, H. C. J. Am. Chem. Soc. 2000, 122, 4217. 